\title{
DESIGN OF A DIGITAL MLSE RECEIVER FOR MOBILE RADIO COMMUNICATIONS
}

\author{
E.Del Re (1) , G.Benelli (2), G.Castellini (3), R.Fantacci (1), L.Pierucci (1), L.Pogliani (4)
}

(1) Universita' di Firenze - Dipartimento di Ingegneria Elettronica

Via S.Marta,3 50139 FIRENZE ITALY

Phone: + 39554796285 Fax: + 3955404392

(2) Universita' di Pavia Via Abbiategrasso,209 27100 PAVIA ITALY

Phone: +39 382391200 Fax:+39382 422881

(3) Istituto di Ricerca sulle Onde Elettromagnetiche - C.N.R

Via Panciatichi,64 50127 FIRENZE ITALY

Phone: + 39554378512 Fax: + 3955410893

(4) O.T.E. S.p.A. Via Barsanti, 8 50127 FIRENZE ITALY

Phone: + 395543811 Fax: +39 554381321

\begin{abstract}
This paper is concerned with a digital receiver suitable for use in the future GSM PanEuropean Cellular system. The digital receiver under consideration employs a maximum likelihood sequence estimation (MLSE) implemented by means of the Viterbi algorithm to compensate for the heavy selective distortions caused by multipath propagation. The performance of receiver is evaluated through a channel simulator suitable for mobile communications. The obtained results show the good behavior of the proposed digital receiver in different modes of operation and an easier implementation in VLSI technology.
\end{abstract}

\section{INTRODUCTION}

Mobile communications are expected to be one of the fastest growing fields in the near future, including terrestrial and satellite communications. A digital land mobile radio system has been recently standardized by the European PTT administrations to be operate in the $900 \mathrm{MHz}$ frequency band.

The main feature of this system is the use of a TDMA scheme in transmission with eight speech channels on the same RF carrier, a gross bit rate of 270.833 kbits/s and Gaussian minimum shift keying (GMSK) modulation.

The TDMA frame is divided in 8 time-slots, each $0.577 \mathrm{~ms}$ long. Each time-slot is reserved to an user to transmit a data packet composed of 148 bits, as shown schematically in Fig. 1. Its physical content is called a burst.

The mobile communication channel is characterized, by fading due to multipath propagation, and by a greater attenuation than the free-space path loss, in addition to noise and other more conventional disturbances. In general the mobile communication channel is better represented by a time-varying model.

The paper is focused on the performance evaluation of an advanced adaptive digital receiver which includes channel decoding operations. In particular the digital receiver considered here makes

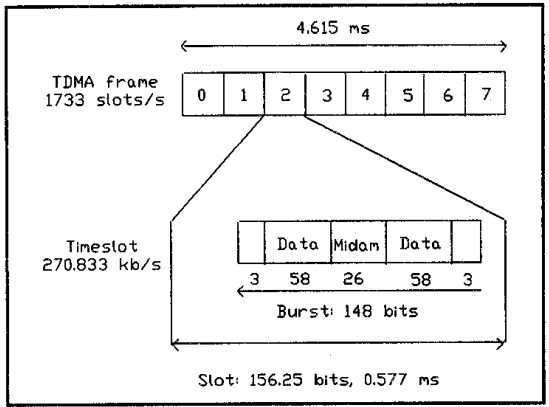

Figure 1 - Time frames, time slots and bursts

use of a maximum likelihood sequence estimation criterium (MLSE) implemented by means of the Viterbi algorithm.

\section{MODEL OF THE MOBILE RADIO CHANNEL}

The propagation of the electromagnetic field between the fixed station and the mobile unit is affected by many factors, including tropospheric scattering, diffraction from natural and artificial obstacles, topographic and environmental conditions. All these factors lead to characterize the signal amplitude received at the mobile unit as composed of two terms :

i) a slow fading component, mainly due to the local topographic conditions, antenna height and other environmental conditions. This slow fading component of the received signal may remain approximately constant along distances of the order of 20 to 30 wavelengths (at least for frequencies below $1 \mathrm{GHz}$ ) and so is not the major concern for the receiver design.

ii) fast fading component, due to the reflections from obstacles and the vehicle movement. This component must be carefully considered in the receiver design. Generally the assumed model for the envelope of the signal affected by this

\subsection{1}


type of fading is the Rayleigh distribution or the Rice distribution.

Due to the multipath propagation, a transmitted impulse signal produces several replicas at the receiver at different time instants. The delay spread measures the time dispersion of the received signal and, in the $900 \mathrm{MHz}$ band, it is typically about $0.1 \mu \mathrm{s}$ on flat terrain, $2 \mu \mathrm{s}$ in urban areas and up to $5 \mu \mathrm{s}$ for hilly terrain. The maximum delay is the delay of the last received significant replica and it can be $0.5 \mu \mathrm{s}$, $10 \mu$ s and $20 \mu$ s respectively [1].

The characteristics of the radio channel can be therefore described by a time-varying impulse response $\mathrm{c}(\tau, \mathrm{t})$, that is a function of the response delay $r$ at the current time t. A common model for the simulation of the fading signal in mobile radio communication is shown in Fig. 2.

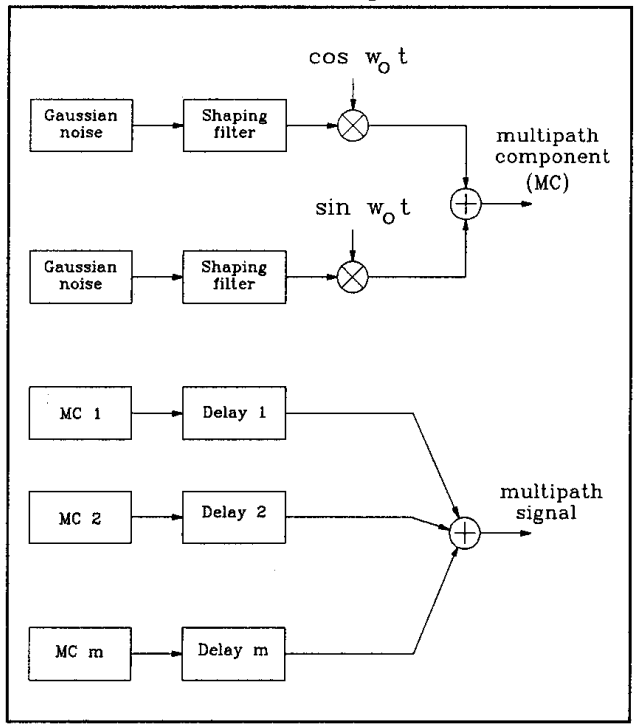

Figure 2 - Mobile radio channel simulator

As example Fig. 3 shows the equivalent lowpass impulse response of the mobile channel in a typical area. The shapes of the impulse responses is different in various propagation conditions and the impulse response has a relatively long duration.

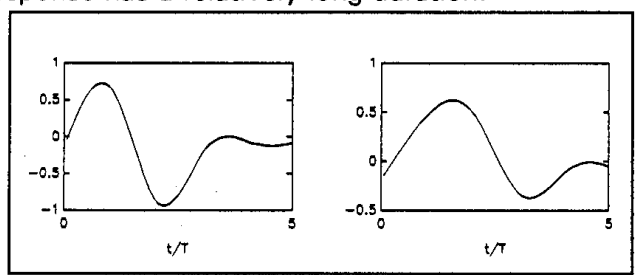

Figure 3 - In-phase and quadrature impulse response (Urban Area $50 \mathrm{Km} / \mathrm{h}$ )

\subsection{Model for digital modulations}

In the pan-European digital cellular system GSM is adopted the Gaussian Minimum shift keying (GMSK) modulation which belongs to the CPM modulation class.

A CPM signal in baseband notation is

$$
s(t)=\exp [j \phi(t, \underline{b})]
$$

where $j=\sqrt{ }-1$

$\underline{b}=\left(\ldots, b_{k}, \ldots\right)$ is the data sequence vector to be transmitted $\phi(t, \underline{b})$ is the modulating phase function

For modulation index $h=0.5$ the CPM signal $s(t)$ can be approximated by the linear QAM signal [2]

$$
x(t)=\sum \exp \left(j \frac{\pi}{2} \Sigma_{k} b_{k}\right) f(t-i T)
$$

$f(t)$ denotes the real valued pulse shaping function. For the GMSK signal with BT $=0.3$ is a Gaussian function spanning the time interval $0 \leq t \leq L T, T$ is the symbol period and $L$ is approximately 3 in the GSM system modulation (without multipath).

Considering $a_{k}=a_{k-1} b_{k}$ the data sequence obtained from $\underline{b}$ by differential encoding the signal $x(t)$ can be simplified

$$
x(t)=\sum_{k} a_{k} j^{k} f(t-k T)
$$

(the symbols +1 and -1 represented the logical 0 and 1 states). So the data symbols are phase-rotated in the complex plane by consecutive multiples of $\pi / 2$. The signal $x(t)$ modulates a carrier frequency, is transmitted through the channel, filtered and translated again to baseband at the receiver.

We can model the received signal at baseband, neglecting the noise term, as

$$
y(t)=\sum_{k} a_{k} j^{k} h(t-k T)
$$

where

$$
h(t)=f(t) * g(t)
$$

and $g(t)$ is the complex lowpass response equivalent of the cascade of transmit filter, channel and receive filter. Therefore $h(t)$ represents the equivalent impulse response of the system from the symbol source to the input of the detector. By applying a "derotation factor" $j^{-k}$ the received signal can be expressed as

$$
y(t)=\sum_{k} a_{k} h(t-k T)
$$

As a consequence of the derotation technique, a simplified receiver structure is obtained. 
Assuming to take one sample of $y(t)$ every $T$ seconds and considering that the impulse response of the radio channel varies with time, a more appropriate model for the sampled baseband signal at the detector input is

$$
y(n T)=\sum_{k} h_{n}(k T) a_{n-k}
$$

where $h_{n}(k T)$ indicates the complex time-varying sampled lowpass equivalent impulse response from source to detector.

\section{A DIGITAL RECEIVER FOR THE GSM SYSTEM}

In the GSM system, the reliability of the digital information is strongly degradated by multipath introduced by the mobile radio communications. Therefore, an accurate design of the receiver structure must be performed. The Maximum Likelihood Sequence Estimation (MLSE) using the Viterbi algorithm seems one of the most powerful method for the equalization of channels with severe distortions.

The general structure of the digital receiver is shown in Fig. 4. The received signal $y(t)$ is reported in the base band and sampled at the converter.

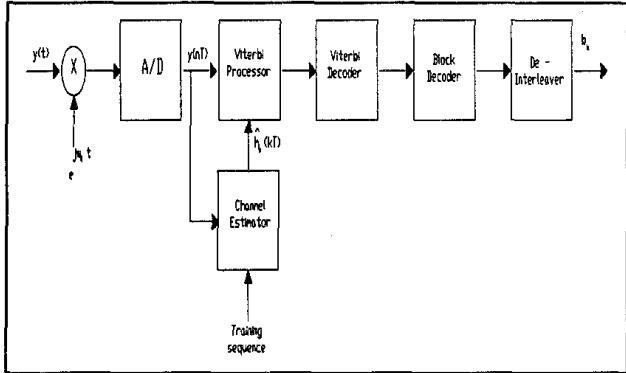

Figure 4 - General receiver structure for the GSM system

Including the noise component, the received signal results

$$
y(n T)=\sum_{k} h_{n}(k T) a_{n-k}+w(n T)=s(n T)+w(n T)
$$

where $w(n T)$ are the samples of an additive noise term. Sequence estimation at a time instant $M$ aims to derive the whole data symbol sequence vector $\mathbf{A}=$ $\left[a_{M} \ldots \ldots . . a_{1}\right]_{T}$ based on the received signal vector (observations) $Y=[\mathrm{Y}(\mathrm{T}) \ldots \ldots \ldots \mathrm{y}(\mathrm{MT})]^{\top}$.

For additive independent Gaussian noise components w(iT), it is known that the MLSE criterion leads to a receiver that has to select among all possible data vectors the vector Â whose corresponding signal vector $\hat{S}$ is closed in Euclidean distance to the observation vector $Y$. In other words it selects the vector Â such that minimizes

$$
\|Y-\hat{S}\|^{2}=\sum_{i=1}^{M}|Y(i T)-\hat{s}(i T)|^{2}
$$

The implementation of the MLSE criterion with the metric (9) can be efficiently performed by the well-known Viterbi algorithm. From the computational point of view, the Viterbi algorithm applied to the MLSE requires at any time instant $i T, i=1, \ldots, M$, the evaluation of the values of the signal component s(iT) for the possible combinations of data symbols according to $(7)$, in order to update the metric calculation. At the end of a block of $M$ received samples, the Viterbi algorithm determines the $M$ data symbols that minimizes $(9)$ and represents the MLSE of the transmitted data symbols. It is not necessary to enter here in further details of the Viterbi algorithm. It is sufficient to point out that:

i) it requires the knowledge or the estimate of the equivalent channel impulse response $h_{n}(k T)$;

ii) $h_{n}(k T)$ must be of the FIR type, say of $\operatorname{order} N$, $\mathrm{k}=0,1, \ldots \mathrm{N}-1$;

iii) the algorithm supplies the MLSE sequence of $M$ data symbols at the end of a received block of information of size $M$, i.e. there is an inherent delay in the detected data sequence.

The MLSE digital receiver consists essentially of two blocks: the channel estimator and the Viterbi processor as shown in Fig. 5.

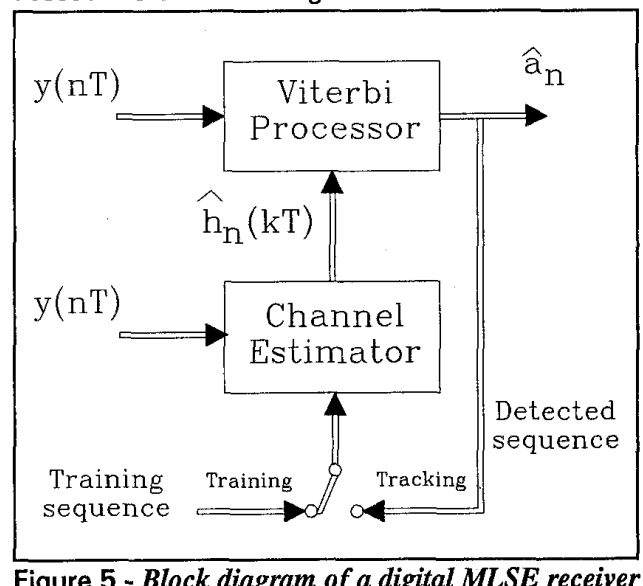

In the GSM system, the data packet contains a pseudo- random sequence of 26 bits, termed midamble, which is known at the receiver. To this regard, the channel estimator evaluates the correlation $C_{i}$ between the samples of the received signal $y(n T)$ and the $N$ symbols $a_{n}$ of the midamble:

\subsection{3}




$$
C_{\digamma}=\frac{1}{N} \sum_{n=0}^{N-1} \sum_{k=-L}^{L} a_{n} a_{n-k+1} h_{k}
$$

Eight different midamble sequences have been selected by the GSM group to optimize the autocorrelation properties.

By using the correlation properties of the midamble it is possible to estimate the coefficients $h_{k}$ of the system response. In the practical implementation of the receiver, the system response have been assumed to span 5 symbols. The Viterbi algorithm uses the estimated coefficients $h_{k}$ to equalize and demodulate the received symbols implementing a trellis structure with 16 states. The Viterbi equalizer evaluates for each path the Euclidean distance between the received sequence and the reconstructed signal using the estimated channel coefficients $h_{k}$, to give at its output the MLSE sequence and a soft information on each demodulated symbol.

This soft information ( 3 bits) which gives an estimate of the data reliability, is used in the next block of the receiver that implements the convolutional decoding based on the Viterbi algorithm.

The suitable use of this information significantly improves the decoder efficiency as to the decoder implementation without the information (hard decision). The side information is obtained together to the calculating of the minimum distance sequence and so is not a considerable amount of the receiver complexity.

Furthermore some other considerations are important from the implementation point of view of the digital receiver.

A) The preamble position in middle of the burst allows to produce a channel impulse response estimate that in many situation is sufficiently accurate for the whole duration of the burst even in presence of time-varying multipath fading. For this reason the Viterbi algorithm is initialized at the extremes of the midamble and then works on the informative sequence in direct and inverse propagation. As expected the performance of the receiver is worse at the beginning and at the end of the burst, and only in the case of high speed of the mobile could be necessary to cope with the fast channel response variations by a receiver adaptability (tracking mode).

B) As long as the equivalent baseband representation (8) of the communication channel is correct, the MLSE receiver only needs the estimate of the channel impulse response $h_{n}(k T)$ and does not require the knowledge of the carrier phase and of the symbol timing. These information are included automatically in the channel estimate $\hat{h}_{n}(k T)$. Therefore the MLSE receiver does not require subsystems dedicated to the carrier phase and symbol timing extraction. Moreover even a moderate frequency offset between the carrier frequency and the frequency of the receiver local carrier can be tolerated. The frequency offset appears as a slowly time-varying relative carrier phase that contributes to the time variations of the channel impulse response.

C) As underlined the value of the parameter $\mathrm{N}$ affects the receiver complexity. However, in practice the receiver of Fig. 5 is robust with respect to the value of $\mathrm{N}$. If turns out that the receiver performance (in terms of error probability) is quite good even for values of $\mathrm{N}$ significantly smaller than the actual duration of the channel impulse response. Moreover it turns out that the digital implementation of the MLSE receiver of Fig 5 requires short binary register for a satisfactory performance, i.e. the receiver is robust with respect to a finite-arithmetic implementation.

The characteristics outlined above suggest that the MLSE receiver is a good candidate for a digital receiver for mobile communications and is suitable for a VLSI implementation.

A structure of the receiver with a more straight forward design and easier implementation in VLSI technology can be obtained using a simplified method for sampling in-phase and quadrature components.

Generally, the all-digital demodulator/detector uses complex sampling which employs double conversion $A / D$ to sample the signal and to produce baseband in-phase (I) and quadrature phase (Q) signals. The analog signal is mixed with quadrature sinusoid at the center frequency $2 \pi f_{o}$ and the outputs are then low-pass filtered to remove the double-frequency terms.

A digital method for sampling in-phase and quadrature components consists to sample the signal at four times the center frequency $4 f_{o}$. This operation supplies alternatively the $I$ and $Q$ data streams by an appropriate multiplication with +1 or -1 [3].

The price of the simplification is that the 1 and 0 samples are obtained at different time instants but this misalignment can be overcame using an interpolation technique on the sequence of the samples.

In our case, the derotation technique has to apply at the received signal and requires to mix down the signal at the IF frequency $f_{\mathrm{IF}}=1 / 4 \mathrm{~T}$. To obtain the above mentioned simple method of sampling it is therefore necessary to sample at the symbol period. This simplified configuration of the receiver with a single $A / D$ converter and the all subsequent processing performed digitally is actually under investigation. 


\section{SIMULATION RESULTS AND CONCLUSION REMARKS}

A computer simulation program has been set up in order to evaluate the performance of a TDMA mobile radio system with the proposed MLSE receiver.

The following assumption will be made:

the normalized bandwidth of the premodulation filter in the GMSK transmitter is BT $=0.3$.

the baseband receiver filter has a $3 \mathrm{~dB}$ band width (two-sided) equal to $180 \mathrm{kHz}$

the receiver structure includes a 16-state Viterbi detector and a 26 bit midamble is used to initialized at the receiver at each time-slot.

The simulated channel impairments are:

flat Gaussian noise

- Rayleigh fading with Doppler frequency shift and multiple echoes selected by the COST Propagation Group as representative of urban area (TU), rural area (RA) and hilly terrain (HT). The error rate performance in absence of channel distortions and in more complex echo patterns is shown in Fig. 6 and in Fig. 7 respectively, as a function of the energy bit/noise spectral density $E_{b} / N_{0}$ Furthermore some considerations of the simulation results are given in the following:

- It can be observed that urban channel is more selective than rural one because includes rather long echo delays.

- It can be noticed that the bit errors is worse at the beginning and at the end of the burst due to the variations of the channel with respect to its estimate at the center of the burst. This indicates that a channel adaptivity during the burst should improve the receiver performance. The simulation results prove that the proposed receiver is not highly sensitive to carrier phase and symbol timing offsets.

- The simulation results prove that the degradation due to a finite-precision implementation of the receiver is acceptable even with an 8-bit arithmetic.

- In the simulation tests also a Viterbi modified detector is implemented to improve the computational efficiency of the algorithm without degrading significantly the general performance of the system.

The metric calculation and the number of comparisons along the Viterbi trellis are reduced rejecting a number of survivor paths according to a threshold criterion.

Fixed a threshold $m$, at each step of the 16 states Viterbi algorithm the $16-\mathrm{m}$ paths having a larger distance from the received sequence are rejected. At the following step, these $m$ survivor paths branch out in $2 \mathrm{~m}$ paths which lead to a number of states included from $m$ to 16 .

The reduction of the number of analyzed states reduces the calculation complexity of the receiver and achieves good detection performance even with $m=8$.

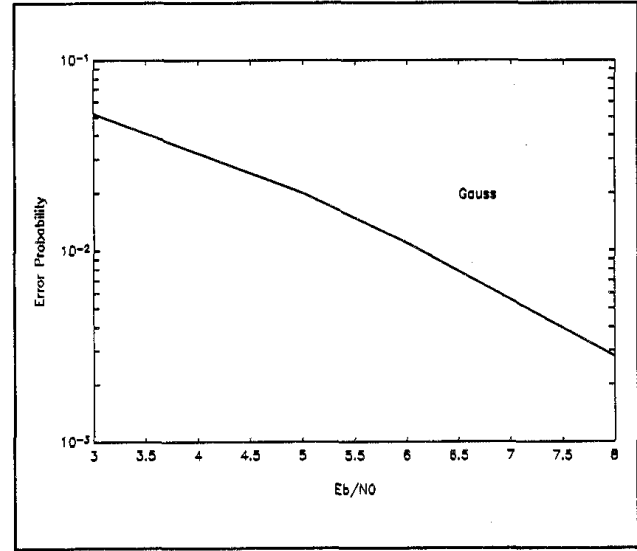

Figure 6 - Bit error rate performance of the receiver on AWGN channel

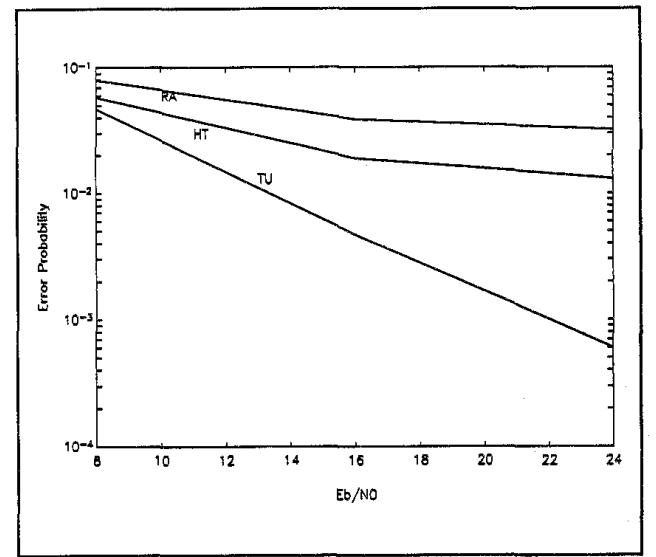

Figure 7 - Bit error rate performance for the receiver on TU - Urban Area, HT - Hilly Terrain, $R A$ - Rural Area with speed of $50 \mathrm{~km} / \mathrm{h}, 100 \mathrm{~km} / \mathrm{h}$ and $250 \mathrm{~km} / \mathrm{h}$ respectively

\section{REFERENCES}

[1] CEPT/CCH/GSM Recommendations.

[2] P. A. Laurent, "Exact and approximate construction of digital phase modulation by superposition of amplitude modulated pulses (APM)", IEEE Trans. Commun., vol. COM-34, pp. 150-160, Feb. 1986

[3] G.J.Saulnier, C.Mcd.Puckette,IV, R. C. Gaus,Jr., R.J. Dunki-Jacobs, T.E. Thiel

"A VLSI demodulator for digital RF network applications: Theory and Results", IEEE Journal on Selected Areas in Communications, Vol. 8, N.8, Oct. 1990.

[4] R. D'Avella, L. Moreno, M. Sant'Agostino, "An adatptive MLSE receiver for TDMA digital mobile radio", IEEE, J. of Selected Areas on Communications, vol. $7, \mathrm{~N}^{\circ} 1$, p.122-129, January 1989. 